\title{
Automatic Seizure Detection in ECoG by Differential Operator and Windowed Variance
}

\author{
Kaushik Kumar Majumdar, Senior Member, IEEE, and Pratap Vardhan
}

\begin{abstract}
Differential operator has long been used in image and signal processing with great success to detect significant changes. In this paper we show that differentiation can enhance certain features of brain electrophysiological signals, contaminated with noise, artifacts, and acquisition defects, leading to efficient detection of those changes. Windowed variance method has been very successful in detecting seizure onset in the brain electrophysiological signals. In this paper we have combined these two powerful methods under the name of differential windowed variance (DWV) algorithm to automatically detect seizure onsets in almost real time, in continuous ECoG (depth-EEG) signals of epileptic patients. The main advantages of the method are simplicity of implementation and speed. We have tested the algorithm on $369 \mathrm{~h}$ of nonseizure ECoG as well as $59 \mathrm{~h}$ of seizure ECoG of 15 epileptic patients. It detected all but six seizures $(91.525 \%$ accuracy) with an average delay of $9.2 \mathrm{~s}$ after the onset with a maximum false detection of three in $24 \mathrm{~h}$ of nonseizure data. Eight novel empirical measures have been introduced to avoid false detections. To ascertain the reliability of the detection method a novel methodology, called quasi-ROC (qROC) curve analysis has been introduced. DWV has been compared with a difference filter based sharp transient (ST) detection algorithm.
\end{abstract}

Index Terms-Automatic seizure detection, differentiation, electrocorticoencephalogram (ECoG), qROC curve, windowed variance.

\section{INTRODUCTION}

D IFFERENTIATION of a function represents its rate of change with respect to its argument. An image is represented as a spatial intensity function. Double differentiation (in the form of Laplacian operator) of the intensity function is widely used for edge detection in an image [1]. An edge can be characterized by an abrupt change in intensity indicating the boundary between two regions of an image [2]. Laplacian has been used to detect the gradient. We have applied a similar logic in this paper to detect the boundary between a significant event, such as an epileptic seizure, and the back ground activity in

Manuscript received September 25, 2010; revised December 25, 2010 and March 18, 2011; accepted May 10, 2011. Date of publication May 27, 2011; date of current version August 10, 2011.

K. K. Majumdar is with the Systems Science and Informatics Unit, Indian Statistical Institute, Bangalore 560059, India (e-mail: kmamjumdar@isibang.ac. in).

P. Vardhan is with the Department of Electronics and Communication Engineering, Maulana Azad National Institute of Technology, Bhopal 462051, India (e-mail: pratapapvr@gmail.com).

Color versions of one or more of the figures in this paper are available online at http://ieeexplore.ieee.org.

Digital Object Identifier 10.1109/TNSRE.2011.2157525 the brain electrophysiological signals. An immediate application of which is automatic detection of seizure onset in the data of a continuously monitored epileptic patient [3]-[5]. It has already been observed that double differentiation of human scalp EEG resulted in marked intensification of fast waves [6]. In fact taking $1.4 \mathrm{~Hz}$ as the threshold point it has been shown that the derivative of the EEG enhances the power associated with frequencies $>1.4 \mathrm{~Hz}$ and at the same time diminishes the power associated with frequencies $<1.4 \mathrm{~Hz}$. From the argument presented in [6] it is evident that the threshold should have been $1 \mathrm{~Hz}$ instead of 1.4. In this paper we will use this phenomenon as a high pass filter with cutoff frequency $1 \mathrm{~Hz}$, which is advantageous for eliminating movement artifacts [7].

First temporal derivative has been used as a filter to enhance the automatic seizure detection accuracy in the ECoG of epileptic patients [3], [5]. Absolute value of first and second derivative of neonatal sleep EEG were used for feature extraction in order to automatically detect the sleep stages [8]. First and second derivative of EEG were also used to extract time domain features for automatic seizure detection in [9]. Normalized absolute value of first or second derivative (depending on the patient) has been used to amplify the seizure part of the depth EEG with respect to the background [10], which facilitated automatic seizure detection (see also [11]). This is in conformity with the use of the differential operator in signal processing in the measurement of small amounts of substances in the presence of large amounts of potentially interfering materials. In such applications it is common that the actual signals are weak, noisy, and superimposed on large background signals [12] from which the actual signals need to be separated out. The biggest advantage of using a differential operator in this context is that it is linear and therefore suitable for online applications [5].

Seizure is often signified by abnormal synchronization in neuronal firing either in a focal region or across several regions in the brain. Synchronous firing during a seizure is largely performed by the excitatory pyramidal neurons (which constitute $85 \%$ of the cortical neurons). As a result sharp spiking activities in quick succession are observed in the excitatory postsynaptic potential (EPSP). Therefore seizure electrophysiological signals both on and inside the scalp are characterized by closely spaced spiking activities relative to the background. Clearly windowed variance will be a simple but efficient method to identify a seizure from the background [13], [14]. Differentiation accentuates the spiking activities and suppresses the background [10], which makes seizure detection by the windowed variance more accurate. This has been utilized for automatic seizure detection in the current work. However this 


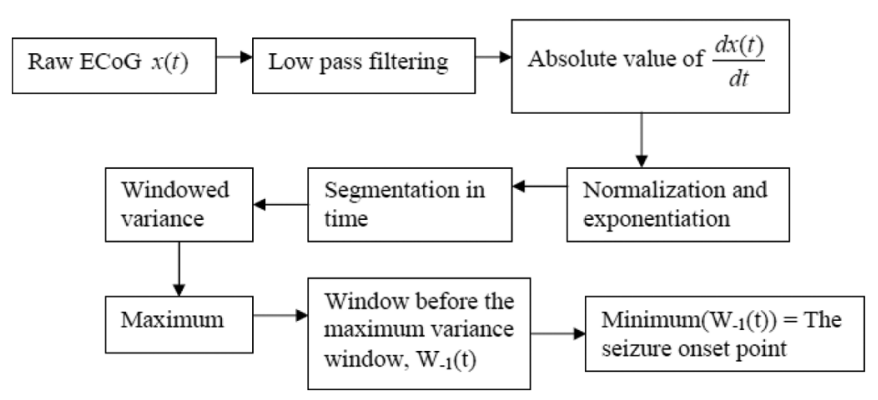

Fig. 1. Schematic block diagram of the DWV algorithm.

also enhances the rate of false detection in the nonseizure part of the signals. To prevent this, eight novel empirical techniques have been introduced, all of which are executable in linear time.

In the next section we will describe the method of seizure detection. In Section III we will describe the methods for prevention of false detection. In Section IV data acquisition will be discussed. Section $\mathrm{V}$ will contain the results of implementation of the methods on ECoG of 15 epileptic patients. Detection performance study by the newly introduced qROC curve analysis will also be presented. In Section VI we have compared differential windowed variance (DWV) with a difference filter based ST detection algorithm that has been described in [3]. We will use EEG and ECoG interchangeably throughout this paper. The last section contains concluding remarks.

\section{Automatic Seizure Detection}

Fig. 1 represents a schematic block diagram of our automatic seizure detection algorithm DWV: $t$ stands for time. The MATLAB code of implementation of the DWV can be downloaded from the first author's website. The DWV algorithm as presented in Fig. 1 has three major subparts, namely enhancement (accentuate the seizure part of the signal compared to the background), filtering (apart from the low pass Gaussian filter that we have used here, DWV also works as a high pass filter), and detection (which actually identifies the seizure onset point). We will be elaborating each of these three parts in the following three subsections.

Here we have used Gaussian low pass filter on the raw ECoG data rather than the more conventional infinite or finite impulse response (IIR or FIR, respectively) low pass filters. When Gaussian filtering is used, more weights are assigned to the particular point where Gaussian filtering is applied and higher weights closer to the same point whereas less and less weights are assigned to the points away from the point of application of the filter. This width of influence is controlled by the variance of the Gaussian [15]. This we have found particularly useful in preserving the distinct shapes of epileptic seizure spikes and sharp waves over the spikes of artifacts.

In case of IIR/FIR, both feed forward and feed back signal points are considered. That is, for applying IIR/FIR at a particular point (say, $n$th point), signal value of the previous points (n-1, n-2, etc., up to the length of IIR/FIR window) one also has to consider the filtered signal (that is output from $\mathrm{n}-1, \mathrm{n}-2$, etc.). This second part is the major difference of the IIR/FIR filters

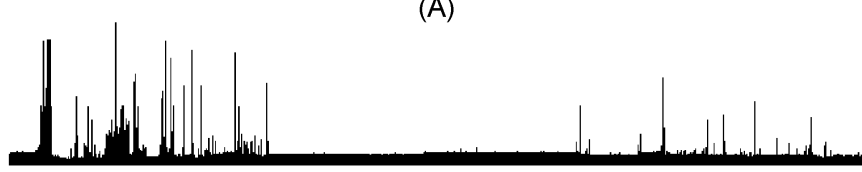

(B)

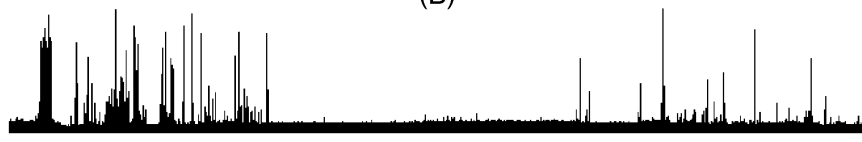

(C)

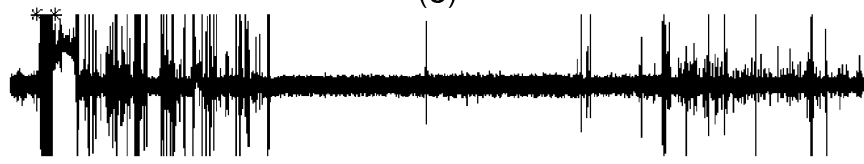

Fig. 2. A $1 \mathrm{~h}$ snapshot of a time domain raw ECoG from a seizure focal channel of patient 4. Epileptologist identified clinical seizure onset and offset have been marked by $*$. The raw signal is full of chewing artifacts $(C)$. Normalized exponential of absolute value of differentiation of the low pass filtered signal at $50 \mathrm{~Hz}$ (B). Normalized exponential of absolute value of double differentiation of the same (A). Onset detection based on (B) is about $7.5 \mathrm{~s}$ closer to the actual onset than that based on (A).

with the Gaussian filter. However it is quite possible to approximate Gaussian filtering with some variations of IIR/FIR filters [15], although the implementation may become slightly more complicated.

\section{A. Enhancement}

DWV achieves seizure features enhancement in a single channel brain electrophysiological signal $x(t)$ (ECoG and EEG) in the following way.

1) $y(t)=G(x(t), f)$, where $G$ is Gaussian (low pass) filter and $f$ is the cutoff frequency.

2) $X(t)=\exp \left((1 / w)\left|D^{\prime} y(t)\right|\right), D^{\prime}$ is the derivative with respect to $t$ and $w$ is a normalization constant (positive and large; in the present work $w=100000$ throughout; it is very important to note that most of the threshold values that we have used in this work are sensitive to $w$ ) [Fig. 2(b)].

Due to the transformation $x \rightarrow X$ as described in 1 and 2, in case $x$ is from the seizure focus in the brain of an epileptic patient, the seizure part of $x$ gets enhanced considerably with respect to the background (which is suppressed to a good extent) in $X$. The reason is very simple. Let $a, b$, and $c$ are successive time points. If a spike occurs at $b$ then statistically $x(b)-x(a)$ and $x(c)-x(b)$ both have high numerical value, which are two successive points in $D^{\prime} x$. On the other hand if all the three points belong to normal background signal then $x(b)-x(a)$ and $x(c)-x(b)$ both will have small values (actually smaller than the average background signal amplitude). Thus $D^{\prime} x$ will enhance the spikes of $x$, but will suppress its background. This particular property of differentiation of the seizure EEG has already been observed in [4]. The effectiveness of 1 and 2 varies across the patient population. For some patients the enhancement in the seizure part may be quite weak, for others it may be pretty sharp. In some cases it may even help nonexperts to identify seizure by visual inspection, which is not so prominent in the raw signal. It can be seen in Fig. 2(a) and (b) how filters based on double differentiation and single differentiation respectively 
have suppressed (to a good extent) the chewing artifacts that are quite prominent in the raw signal [Fig. 2(c)].

\section{B. High Pass Filter}

Fourier expansion of $X$ can be written as

$$
X(t)=\frac{a_{0}}{2}+\sum_{n=1}^{\infty}\left(a_{n}^{2}+b_{n}^{2}\right)^{1 / 2} \sin \left(\frac{2 \pi n t}{p}+\alpha_{n}\right)
$$

where $a_{n}$ is Fourier coefficient associated with each frequency component $n, p$ is the period of the signal and $\alpha_{n}$ is the phase associated with $n$ given by $\alpha_{n}=\tan ^{-1}\left(\left(a_{n} / b_{n}\right)\right)$ [16]. Although EEG is never periodic (nonstationary), but if its Fourier expansion is assumed to be valid then (1) must have to be true for some $p$ [17, Ch. 11]. Differentiating (1) we get

$$
X^{\prime}(t)=\left(\frac{2 \pi}{p}\right) \sum_{n=1}^{\infty} n\left(a_{n}^{2}+b_{n}^{2}\right)^{1 / 2} \cos \left(\frac{2 \pi n t}{p}+\alpha_{n}\right)
$$

where $X^{\prime}$ is the derivative of $X$. If right side of (1) is to converge to the left then $\left(a_{n}, b_{n}\right) \rightarrow(0,0)$ as $n \rightarrow \infty$. Also $p \gg 2 \pi$. So components for small values of $n$ tend to vanish. Therefore (2) acts as a high pass filter with respect to a phase shift of $\pi / 2$ (a $\pi / 2$ phase shift preserves many features of a signal including the spike trains). We can reasonably assume that it is a high pass filter with cutoff frequency $n=1$ (also see [6]).

\section{Detection}

It has been observed that the windowed variance is one of the most efficient methods to detect changes in an EEG or ECoG signal due to epileptiform activities [13], [14]. At the same time it is a linear method. We calculate $V_{W}(X(t), l, s)$, where $V_{W}$ is the windowed variance operation with window length $l$ and sliding distance $s$. The windowed variance graph $\mathrm{Wvar}(t)=$ $V_{W}(X(t), l, s)$ has been shown in Fig. 3 with peak onset and offset clearly identified as local minimum before and after the seizure peak respectively. The seizure peak has been automatically identified as the maximum value of $\mathrm{W} \operatorname{var}(t)$ in a 1-h-long snapshot of continuous stream ECoG data. Once a peak is identified as a seizure it is marked and never taken into consideration for further identification. The process continues to search for seizure peak after the last identified one. Please note that although the seizure peak identification is in real time, the actual seizure onset detection is slightly behind the real time. We will see in Section V that in almost all cases that have been studied the computer identified onset is very close to the epileptologist identified onset (Fig. 2).

\section{Avoidance of False Detection}

The more efficient a seizure detection method the higher is the chances of false seizure detection in the nonseizure data. In order to eliminate false detection as far as possible we have introduced eight novel empirical tests as following. To be qualified as a seizure the data must be identified as having seizure by each of the eight tests separately. Otherwise it will be classified as a nonseizure.

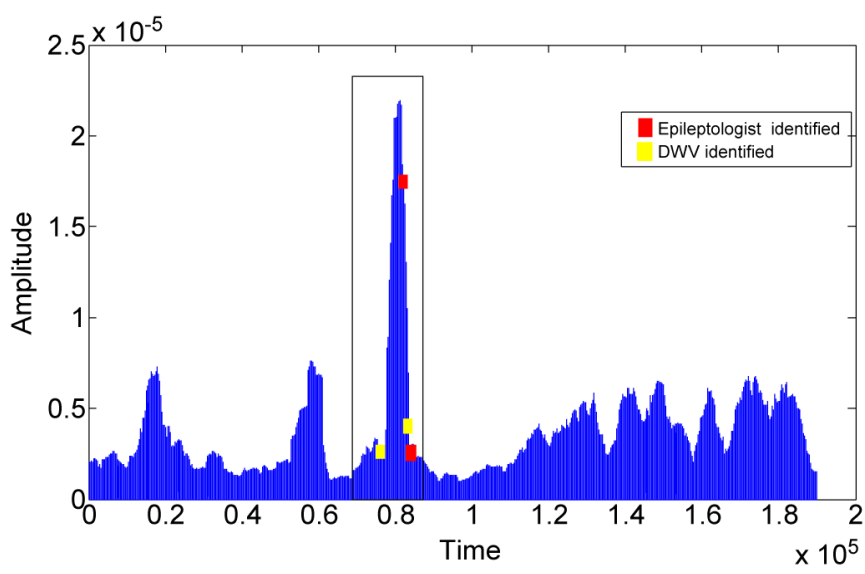

Fig. 3. A $W \operatorname{var}(t)$ snapshot of patient 1 during the seizure at the 13th hour of recording at channel 1 , where the seizure pillar (enclosed in the rectangular box) is distinctly visible. Epileptologist identified seizure onset and offset points are 82010 and 83855 , respectively, and those by DWV are 76802 and 83702 , respectively. Seizure onset and offset time points are indentified by the verical arms of the small solid retangles (right arm for the left most yellow rectangle and left arm for all other rectangles), red for epileptologist indentified points and yellow for DWV identified points. Abscissa denotes time in second $(256$ points $=1 \mathrm{~s})$ and ordinate denotes normalized amplitude.

\section{A. Maximum Windowed Variance on Differentiated Signal}

The windowed variance operation is to be performed on $X(t)$ for fixed window length (across all patients, we have taken it to be 4000 time points, i.e., $4000 / 256=15.625 \mathrm{~s}$ long) and fixed sliding distance (across all patients, we have taken it to be 100 time points long). We denote the output of this operation as Wvar $(X(t))$ or more simply Wvar $(t)$. In the DWV block diagram in the Section II, we have taken "Segmentation in time" $=1 \mathrm{~h}$. The Maximum windowed variance over a 1-h-long $X(t)$ is denoted by $\mathrm{B}=\max (\mathrm{W} \operatorname{var}(t))$ (barring one or two exceptions we are following the same notations and symbols in this paper that we have used in our MATLAB programs that are available online in the first author's website). For a given patient if $B$ is below certain threshold (to be determined by sample seizure data of the patient) then it is a nonseizure. Otherwise it is a seizure. In fact we have also observed that the value should lie not only above a threshold, but below another threshold. If it goes above the latter threshold it is most likely to be an artifact rather than a seizure. So ideally B should lie in an interval (patient specific).

\section{B. Maximum Windowed Variance on Undifferentiated Signal}

Let us take $y(t)$ as in point 1 of Section II-A. Maximum windowed variance operation has been performed on the absolute value of $y(t)$ with the same window length and sliding distance as in Section II-A across all the patients. The variance of the window of maximum variance has been denoted by $\mathrm{C}$. If $\mathrm{C}$ falls below certain threshold (to be determined by sample seizure data of the patient) then it is a nonseizure. Otherwise it is a seizure. Just for the same reasons as in Section III-A in case of a seizure $\mathrm{C}$ should lie within a patient specific interval.

\section{Window After the Maximum Variance Window}

We take $W v a r(t)$ as in Section III-A. Let us denote the window of maximum variance $\mathrm{B}$ by $W_{0}$. In the same vain we 
can denote the window immediately after $W_{0}$ as $W_{1}$. Let $m m$ be an array of variance values of successive windows. Let us write $m m(1)=\operatorname{var}\left(W_{1}\right)$, where $\operatorname{var}()$ denotes the variance. Let us consider $|B-m m(1)|$. If $|B-m m(1)|$ falls below certain threshold (to be determined by sample seizure data of the patient) then it is a nonseizure. Otherwise it is a seizure. Here too for seizure the value should lie within a patient specific interval.

\section{The 3/4th Rule}

Let $m m(i)$ be the variance of $X(t)$ at the $i$ th window $W_{i}$ after $W_{0}$. Number of windows to be considered after $W_{0}$ is to be stipulated beforehand (we typically kept it at 16). Let $n 1(1)$ be the position (taken to be the first or starting point of the window) of the first of the windows with minimum variance among the stipulated number of windows. Let $M$ is the position of $W_{0}$ and $N 1=M+$ (window length) $* n(1)$. Local minimum of $X(t)$ within $W_{-1}$ has been treated as the onset of seizure in this paper (Fig. 3) and local minimum of $X(t)$ within the window $W_{1}$ is the offset (the bottom right panel of Fig. 3). In general we have noticed that this onset is quite satisfactory, but the offset is not that up to the mark. Therefore we are not going to report the offset results in this paper. However it has important role in distinguishing between seizure and nonseizure peaks as we will see in Section III-H below. Let $E$ be an array consisting of values of $\mathrm{W} \operatorname{var}(t)$ starting from two windows before $M$, i.e., $W_{-2}$ through $W_{n 1(1)}$. Let $z$ is an array consisting of maximum values of $E$. Let $F$ be another array consisting of values of $E$ which are greater than or equal to $(3 / 4) \max (E)$. We have noticed that $|\lfloor\operatorname{mean}(F)-z(1)\rfloor|$ is a quantity whose threshold distinguishes between seizure and nonseizure EEG. If it lies below certain threshold then it is a seizure. Otherwise it is a nonseizure. Let us mention that compared to the other thresholds described in Sections III-A to III-C this threshold took fewer different values across the patient population that we have tested. In other words this threshold has remained more uniform across the patient population.

\section{E. The Above 3/4th Rule}

$\lfloor\operatorname{std}(F)\rfloor$, where $s t d$ stands for standard deviation, is a quantity whose patient specific threshold will distinguish between seizure and nonseizure signal windows. There is no seizure if the value is greater than the threshold. This threshold also remained more uniform across the patient population that we have tested compared to the thresholds in Sections III-A to III-C.

\section{F. Double Derivative of $E(D D E)$ Rule}

Let $D E=\exp \left((1 / v)\left|D^{\prime \prime} E\right|\right)$, where $D^{\prime \prime}$ denotes double differentiation and $v$ is a positive normalization constant (kept fixed across all the patients). $E$ is as in Section III-D. Let $K$ be an array consisting of values of $D E$, which are greater than or equal to $0.999 * \max (D E)$. Length of $K$ is a quantity, whose threshold distinguishes between seizure and nonseizure EEG. Greater than threshold length indicates nonseizure. This threshold is also more uniform across the patient population.

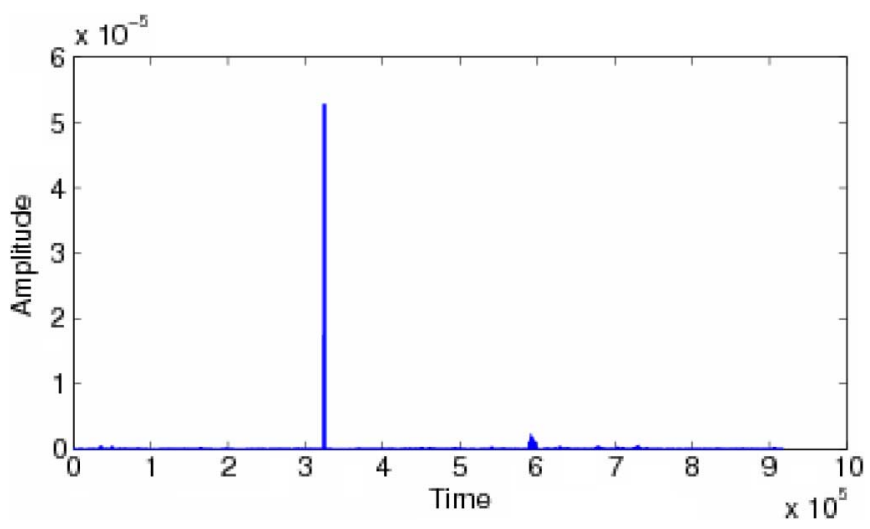

Fig. 4. $X(t)$ form (see Section II-A, point 2) of a 1-h-long seizure ECoG $x(t)$. The seizure peak appears distinctively as a short thick dense pillar above the time point $6 \times 10^{5}$. A tall thin nonseizure peak appears between $3 \times 10^{5}$ and $4 \times 10^{5}$, which is to be eliminated by the peak rejection method. 256 time points $=1 \mathrm{~s}$.

\section{G. Maximum DE (MDE) Rule}

For seizure EEG $\max (D E)$ must lie within a specified interval. This interval is patient specific.

\section{H. Peak Rejection}

The difference between the seizure onset and offset points as described in Section III-D gives the width of a seizure peak or pillar. In case of some patients' data where there are too many noisy spikes this method will help eliminate nonseizure spikes from the signal, thereby making the detection more accurate (Fig. 4). The noise spikes are usually less wide than the seizure spikes (Fig. 4). So if the peak width is less than the threshold it is taken to be a nonseizure peak. Otherwise it is a seizure peak.

It is to be noticed that each of the above operations executes in linear time. The whole method is extremely fast-takes less than $4 \mathrm{~s}$ for a 1-h-long snapshot of continuous stream data, with 15.625-s window length and 15.234 s overlap on an Intel Core 2 Duo Processor T8100 (2.1 GHz/800 MHz FSB, 3M L2 cache), Ubuntu machine with 4 GB RAM. The implementation was in MATLAB (in C++ code the program is likely to run even faster). In a much slower Windows Vista based machine the operations did not take more than $15 \mathrm{~s}$.

Both the DWV and the false detection avoidance rules have been incorporated in the same MATLAB program to run serially one after the other on any single channel continuous ECoG signal (parallel implementation on multi-channel data has also been achieved). First DWV identifies a window and a point of onset of a seizure, irrespective of whether the signal actually contains a seizure or not. Then each of the false detection avoidance rules is run on the signal. If each of them confirms that the identified window then only it is accepted as a seizure window and the onset is calculated as described in Section II.

\section{DATA ACQUISITION}

To test the effectiveness of the DWV algorithm on brain electrophysiological signals along with the false detection avoidance rules, we have chosen to run our algorithm on the publicly available ECoG data from the Seizure Prediction Project of the Albert-Ludwig-Universitat Freiburg, Germany [18]. The ECoG data were acquired using Neurofile NT digital video EEG 
TABLE I

PATIENT DetaILS

\begin{tabular}{|c|c|c|c|c|c|c|c|}
\hline Patient & Sex & Age & $\begin{array}{c}\text { Seizure } \\
\text { Type }\end{array}$ & H/NC & Electrode & Origin & $\begin{array}{c}\# \\
\text { seizures }\end{array}$ \\
\hline 1 & $\mathrm{~F}$ & 15 & $\mathrm{SP}, \mathrm{CP}$ & $\mathrm{NC}$ & $\mathrm{g}, \mathrm{s}$ & Frontal & 4 \\
\hline 2 & $\mathrm{M}$ & 38 & $\mathrm{SP}, \mathrm{CP}, \mathrm{GTC}$ & $\mathrm{H}$ & $\mathrm{D}$ & Temporal & 3 \\
\hline 3 & $\mathrm{M}$ & 14 & $\mathrm{SP}, \mathrm{CP}$ & $\mathrm{NC}$ & $\mathrm{g}, \mathrm{s}$ & Frontal & 5 \\
\hline 4 & $\mathrm{~F}$ & 26 & $\mathrm{SP}, \mathrm{CP}, \mathrm{GTC}$ & $\mathrm{H}$ & $\mathrm{d}, \mathrm{g}, \mathrm{s}$ & Temporal & 5 \\
\hline 5 & $\mathrm{~F}$ & 16 & $\mathrm{SP}, \mathrm{CP}, \mathrm{GTC}$ & $\mathrm{NC}$ & $\mathrm{g}, \mathrm{s}$ & Frontal & 5 \\
\hline 6 & $\mathrm{~F}$ & 31 & $\mathrm{CP}, \mathrm{GTC}$ & $\mathrm{H}$ & $\mathrm{d}, \mathrm{g}, \mathrm{s}$ & Temporo/Occipital & 3 \\
\hline 8 & $\mathrm{~F}$ & 32 & $\mathrm{SP}, \mathrm{CP}$ & $\mathrm{NC}$ & $\mathrm{g}, \mathrm{s}$ & Frontal & 2 \\
\hline 11 & $\mathrm{~F}$ & 10 & $\mathrm{SP}, \mathrm{CP}, \mathrm{GTC}$ & $\mathrm{NC}$ & $\mathrm{g}, \mathrm{s}$ & Parietal & 4 \\
\hline 12 & $\mathrm{~F}$ & 42 & $\mathrm{SP}, \mathrm{CP}, \mathrm{GTC}$ & $\mathrm{H}$ & $\mathrm{d}, \mathrm{g}, \mathrm{s}$ & Temporal & 4 \\
\hline 13 & $\mathrm{~F}$ & 22 & $\mathrm{SP}, \mathrm{CP}, \mathrm{GTC}$ & $\mathrm{H}$ & $\mathrm{d}, \mathrm{s}$ & Temporo/Occipital & 2 \\
\hline 14 & $\mathrm{~F}$ & 41 & $\mathrm{CP}, \mathrm{GTC}$ & $\mathrm{H}$ and & $\mathrm{d}, \mathrm{s}$ & Fronto/Temporal & 4 \\
\hline 15 & $\mathrm{M}$ & 31 & $\mathrm{SP}, \mathrm{CP}, \mathrm{GTC}$ & $\mathrm{HC}$ and & $\mathrm{d}, \mathrm{s}$ & Temporal & 4 \\
\hline 17 & $\mathrm{M}$ & 28 & $\mathrm{SP}, \mathrm{CP}, \mathrm{GTC}$ & $\mathrm{NC}$ & $\mathrm{S}$ & Temporal & 5 \\
\hline 19 & $\mathrm{~F}$ & 28 & $\mathrm{SP}, \mathrm{CP}, \mathrm{GTC}$ & $\mathrm{NC}$ & $\mathrm{S}$ & Frontal & 4 \\
\hline 20 & $\mathrm{M}$ & 33 & SP,CP,GTC & $\mathrm{NC}$ & $\mathrm{d}, \mathrm{s}$ & Temporo/Parietal & 5 \\
\hline
\end{tabular}

$\mathrm{SP}=$ simple parietal, $\mathrm{CP}=$ complex parietal, $\mathrm{GTC}=$ generalized tonic-clonic, $\mathrm{H}=$ hippocampal, $\mathrm{NC}=$ neocortical. Electrode: grid (g), strip (s), depth (d). Seizure frequency varies between 0.1 and 6.8 per day [21, Table I].

system (It-med, Usingen, Germany) with 128 channels, $256 \mathrm{~Hz}$ sampling rate, and a 16 bit analog to digital converter. In all cases the ECoG from only six sites have been analyzed. Three of them from the focal areas and the other three from out side the focal areas (this is the configuration with which the data was available to us). See Table I for the patient details. The patient population have earlier been studied in [19]-[21], where further details can be found.

There are 21 medically intractable focal epileptic patients' ECoG data [18]. For each patient there are $2-5 \mathrm{~h}$ of seizure data and 24-26 h of nonseizure data (except patient 2, for whom no nonseizure data is available). We have tested our algorithm on all of them. Only on 15 of the patients it worked satisfactorily and in this paper we are going to report results on these fifteen patients only. Excluding some patients' data from the actual experiment for various reasons is not uncommon as can be seen in [5]. The six patients' data on which our methods did not work satisfactorily had one or more of the following problems. Data contained heavy artifacts. Because of amplifier box disconnection and reconnection there were sharp jumps in voltage, which have been identified as "seizure" by the DWV and also could not be identified as nonseizure (i.e., not clinical seizure) by the false detection avoidance rules of Section III. For patients 10 and 21's seizure data the authors could not find any seizure spikes by visual inspection.

Patient specific performance of the automatic seizure detection algorithms is well recognized [22], [23]. Since the patient data we have used here is an open source data, we will maintain the patient number, so that the future workers get a chance to compare with our findings.

\section{RESULtS}

\section{A. Preprocessing}

Gaussian low pass filter, with cut off frequency $100 \mathrm{~Hz}(50 \mathrm{~Hz}$ for patient 4 , whose data contains high concentration of chewing artifacts [see Fig. 2(c)] has been used to remove muscle contrac-
TABLE II

Detail of the Patient SPECIFIC Results

\begin{tabular}{|c|c|c|c|c|c|}
\hline $\begin{array}{c}\text { Patient } \\
\text { number }\end{array}$ & Montage & $\begin{array}{c}\text { Recorded } \\
\text { ECoG in } \\
\text { hour }\end{array}$ & $\begin{array}{c}\# \\
\text { seizure } \\
\text { occurred }\end{array}$ & $\begin{array}{c}\# \\
\text { seizure } \\
\text { detected }\end{array}$ & $\begin{array}{c}\# \text { false } \\
\text { positive }\end{array}$ \\
\hline 1 & Unipolar & 28 & 4 & 4 & 0 \\
\hline 2 & Bipolar & 3 & 3 & 3 & N/A \\
\hline 3 & Unipolar & 29 & 5 & 5 & 0 \\
\hline $4(50 \mathrm{~Hz})$ & Bipolar & 29 & 5 & 4 & 0 \\
\hline 5 & Bipolar & 29 & 5 & 5 & $5(2), 2(3)$ \\
\hline 6 & Unipolar & 27 & 3 & 2 & 0 \\
\hline 8 & Unipolar & 26 & 2 & 2 & 0 \\
\hline 11 & Bipolar & 28 & 4 & 4 & 0 \\
\hline 12 & Bipolar & 29 & 4 & 4 & 1 \\
\hline 13 & Bipolar & 26 & 2 & 1 & 3 \\
\hline 14 & Bipolar & 28 & 4 & 3 & 0 \\
\hline 15 & Bipolar & 28 & 4 & 3 & $3(2), 0(3)$ \\
\hline 17 & Unipolar & 29 & 5 & 5 & 0 \\
\hline 19 & Bipolar & 28 & 4 & 3 & 0 \\
\hline 20 & Unipolar & 31 & 5 & 5 & $2(3)$ \\
\hline
\end{tabular}

For Patient 4, the cutoff frequency in the Gaussian filter is $50 \mathrm{~Hz}$, for all other it is $100 \mathrm{~Hz}$. For Patient 2, no nonseizure data is available. The number in bracket in the last column indicates channel threshold.

tion artifacts and noise. Since differentiation has been applied, it worked as a high pass filter with cutoff frequency $1 \mathrm{~Hz}$. The phase shift keeps the seizure part intact. For each of the 15 patients we have tested the data of common reference montage as well as bipolar montage. In a patient specific way one gives better results over the other (Table II), and we have opted for the better in each case. The bipolar montage has been indicated as c1-c2, c2-c3, c3-c1, where $\mathrm{c} 1$ is channel 1, etc. (only channels 1,2 , and 3 are associated with seizure focus).

\section{B. Seizure Detection}

In this paper we are only concerned about the automatic detection of the clinical seizure onset (CSO). The gold standard of CSO is the seizure onset identification by a certified epileptologist. The data that have been provided to us by the Albert-Ludwig-Universitat Freiburg, came with the CSO and the 
TABLE III

Patient-Wise Sensitivity, Specificity, Percentage Detection, AND ERror Rate

\begin{tabular}{|c|c|c|c|c|c|c|c|c|c|c|c|c|c|c|c|}
\hline Patient & 1 & 2 & 3 & 4 & 5 & 6 & 8 & 11 & 12 & 13 & 14 & 15 & 17 & 19 & 20 \\
\hline Sensitivity & 1 & 1 & 1 & 0.8 & 1 & 0.67 & 1 & 1 & 1 & 0.67 & 0.75 & 0.75 & 1 & 0.75 & 1 \\
\hline Specificity & 1 & N/A & 1 & 1 & 0.92 & 1 & 1 & 1 & 0.96 & 0.88 & 1 & 1 & 1 & 1 & 0.92 \\
\hline \% detection & 100 & 100 & 100 & 80 & 100 & 67 & 100 & 100 & 100 & 67 & 75 & 75 & 100 & 75 & 100 \\
\hline \% error rate & 0 & 0 & 0 & 20 & 0 & 33 & 0 & 0 & 0 & 33 & 25 & 25 & 0 & 25 & 0 \\
\hline
\end{tabular}

clinical seizure termination (CST) points identified by certified epileptologists. In all automatically detected seizures but one the CSO detection by the DWV has been achieved within $20 \mathrm{~s}$ of the CSO identified by epileptologist. Only for the seizure of 46th hour of the patient 8 the detection by DWV took place $66.3867 \mathrm{~s}$ after the CSO identified by the epileptologist. Therefore we have considered a CSO detection is successful by the DWV only if the detection by DWV occurs within $67 \mathrm{~s}$ of the epileptologist identified onset (in [5] this has been taken to be $2 \mathrm{~min}$ ).

The detection algorithm was run on 1-h snapshots of ECoG from the epileptic patients containing both seizure and nonseizure signatures (most of them did not contain any seizure and the challenge was to avoid false detections on those data). Window length of 4000 time points (15.625 s) with 3900 (15.234 s) time points overlap (i.e., sliding by 100 points) has been used $(256$ points $=1 \mathrm{~s})$. Seizure portions were identified by certified epileptologists at the place of origin of the data at each given time slot, but not for individual channels. The algorithm was implemented on each of $\mathrm{c} 1, \mathrm{c} 2$, and $\mathrm{c} 3$, or $\mathrm{c} 1-\mathrm{c} 2$, $\mathrm{c} 2-\mathrm{c} 3$, and $\mathrm{c} 3-\mathrm{c} 1$ to automatically detect the onset and the offset of a seizure (please see Table II). Onset of the seizure has been taken to be the earliest point detected as the onset among all the three channels (same has been followed in [5]) or the three channel pair subtractions, as the case may be. In this paper we have identified the offset for each channel or channel pair subtraction for only one purpose i.e., for determining the spike width (as shown in Section III-H) in order to distinguish seizure spikes from the nonseizure spikes (the width may not be accurate all the time, but it nicely serves our purpose of seizure nonseizure spike train distinction).

The single differentiation operation (SDO) has been applied on all patients. For the application of double differentiation operation (DDO) see [10]. SDO works better with more noisy data. In the ECoG of patient 1 windowed variance could not detect seizures in the preprocessed signals. But after filtering with the SDO all the seizures could be detected by windowed variance (in this case DDO performed even better as can be seen in Fig. 1 of [10]). Out of total 59 seizures tested in 15 patients, 54 could be detected accurately $(91.525 \%$ accuracy, which is comparable with [24]), with a total of eight false detections in $369 \mathrm{~h}$ of nonseizure data belonging to the 15 patients (Table II). Patient specific sensitivity, specificity, detection rate, and error rate have been given in Table III.

We do not have any nonseizure data for the Patient 2. Patient 4's data are heavily contaminated with chewing artifacts [Fig. 2(c)]. We have kept the cutoff frequency of the Gaussian low pass filter for this patient at $50 \mathrm{~Hz}$ (this gives better detection than $100 \mathrm{~Hz}$, because chewing artifacts are high frequency artifacts). For all other patients it has been $100 \mathrm{~Hz}$. The fifth seizure ECoG of patient 4 required a very special preprocessing (sup- pression of all values $\geq 0.15$ of the maximum value irregardless of sign) after which the seizure could be detected accurately. But this does not work for other seizures of the same patient. Therefore we will treat it as a failure of the DWV. For patient 5 there are five false detections if channel threshold is 2 , but there are only two false detections if the channel threshold is 3 (Table II). This means if a seizure is detected it will have to be detected in not just 1 single focal channel but in 2 to (all) 3 focal channels (almost) at the same time. When, detection is recognized only if it occurs almost simultaneously at 2 of the focal channels, we call the channel threshold 2 . Similarly for channel threshold 3.

For some patients $X(t)$ is full of nonseizure spikes. One way to efficiently detect the seizure spikes is to eliminate the nonseizure spikes. For this we have developed a peak rejection method (Section III-H). It has been observed that seizure peaks have distinctive pillar like shape compared to much slender artifact peaks in the $X(t)$ signals of the patients (Fig. 4). A peak is rejected if it is a spike window having lower width than a subsequent much wider peak window. A wider peak window typically turns out to be a seizure window. Peak rejection method has been applied only on the data that are heavily contaminated with spike artifacts (ECoG of patients 6, 13, and 14).

Since the available seizure ECoG was rather scarce for each patient, thresholds were set at the time of detection. Then the effectiveness of the method was tested by the number of false positives on the 24 or more hour long seizure free signals. Performance measure has been given in terms of the area under the qROC curve in the next subsection. The average seizure detection time lag is $9.2 \mathrm{~s}$ after the epileptologist determined onset, which is $9.3 \mathrm{~s}$ in [23].

\section{Robustness}

DWV together with the false detection avoidance measures has been successful to automatically detect seizures in 15 out of 21 patients satisfactorily. Out of these 15 patients the data of patients $4,6,13$, and 14 contain heavy artifacts. For patient 4 high-frequency muscle contraction artifacts due to chewing [Fig. 2(c)] have been suppressed to some extent by Gaussian low pass filter with cutoff frequency $50 \mathrm{~Hz}$. DWV tended to identify artifacts as seizure, but false detection avoidance methods helped them to identify as nonseizure artifacts. For patient 4 it was helpful to detect seizures by taking shorter segment signal (30 min instead of $1 \mathrm{~h}$ ) during one particular hour. Also amplitude suppression tended to improve automatic detections when high amplitude artifacts were present (we did not report results on amplitude suppression in this paper). The data of patients 6,13 , and 14 contain plenty of nonseizure spike artifacts, which had to be identified by peak rejection method (Section III-H, Fig. 4) for DWV to successfully detect 
TABLE IV

Patient-Wise False Positive Rate (FPR) and True Positive Rate (TPR)

\begin{tabular}{|c|c|c|c|c|c|c|c|c|c|c|c|c|c|c|c|}
\hline Patient & 1 & 2 & 3 & 4 & 5 & 6 & 8 & 11 & 12 & 13 & 14 & 15 & 17 & 19 & 20 \\
\hline FPR & 0 & $1 / 8$ & 0 & 0 & $1 / 12$ & 0 & 0 & 0 & $1 / 25$ & $1 / 8$ & 0 & 0 & 0 & 0 & $1 / 13$ \\
\hline TPR & 1 & 1 & 1 & $4 / 5$ & 1 & $2 / 3$ & 1 & $3 / 4$ & 1 & $1 / 2$ & $3 / 4$ & $3 / 4$ & 1 & $3 / 4$ & 1 \\
\hline
\end{tabular}

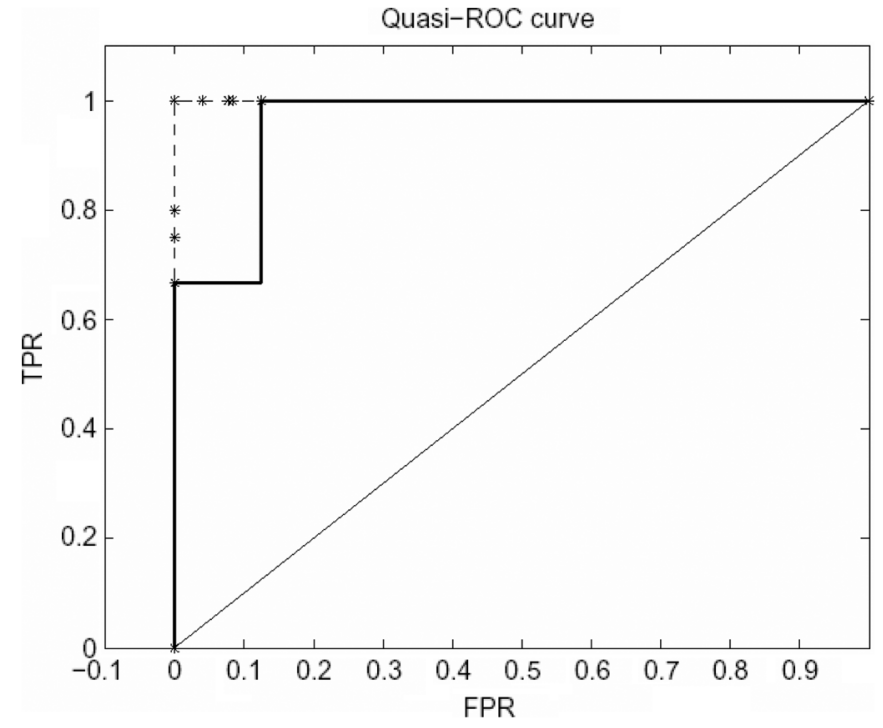

Fig. 5. The quasi-ROC curve (the thick black curve) of seizure detection. $\mathrm{TPR}=$ true positive rate and FPR $=$ false positive rate (values are in Table IV). Data points are denoted by “*." There are multiple data on some points. $(0,0)$ and $(1,1)$ have been taken as the starting and the ending points of the curve. The dimension of the rectangle $\mathrm{R}$ encompassing all the data points is $(1 / 3) *(1 / 8)$. The area under the quasi-ROC curve is 0.9583 .

seizures. However DWV along with the false detection avoidance methods did not work for the six patients out of the total 21 whose data we have tested. We can conclude that for some patients the methods of Sections II and III work excellently (such as patients 1,2, and 3), but for some others they may fail on a significant portion of the data (such as patient 18). Given the fact that seizure characteristics vary widely across the patients and there may even be significant variations in the same patient over the time, this is not unexpected. In fact we have always tested our method on 1-h-long data segment, which is unusually long in the seizure detection research [14], [22]. We have done so to test our method in presence of very long trains of artifacts. 15 out of 21 patients it worked satisfactorily, which indicates good patient specific robustness of the method (both automatic detection and false alarm avoidance methods taken together).

\section{Quasi-ROC Curve Analysis}

ROC curve analysis is a simple, efficient and widely used tool to evaluate the performance of binary classifiers like seizure detectors. In order to compute an ROC curve one needs to have either a genuine probability distribution function (pdf) or some kind of a probability score [25] with which the classifier will classify an object or instance into either of the two classes. However a pdf or a probability score may not always be available for a classifier. In such cases thresholding with respect to the probability score (probability measure is also a probability score), which is essential for generating an ROC curve [25], does not work. This is exactly the situation with the seizure detection (seizure-no seizure classification) in this work. We do not have a probability score to sort the instances in increasing order with respect to that score and therefore cannot generate an ROC curve per se. Also our dataset consisting of only 15 patients is too small a sample to infer a distribution. Here we introduce a new concept called quasi-ROC curve or qROC curve which can evaluate the performance of a classifier without associating any pdf or probability score with it. In fact to generate a qROC curve no notion of probability is necessary.

To evaluate the performance of the DWV along with the false detection avoidance measures, we classify the automatic detections into two classes, positive and negative, which can then be categorized into four subclasses of detections: $\mathrm{TP}=$ true positive, $\mathrm{TN}=$ true negative, $\mathrm{FP}=$ false positive, and $\mathrm{FN}=$ false negative. A detection is considered TP if an actual seizure has been detected (in one or more focal channels as the case may be) within $67 \mathrm{~s}$ of onset (see Section V-B for detail). Detection of a seizure on a nonseizure signal (where all the eight parameters described in Section III have failed to identify it as a nonseizure) has been classified as FP. If a seizure did not occur and also it had not been detected then it is classified as TN. If a seizure occurred yet it had not been detected then it is classified as FN [26].

All this has been summarized in the quasi-ROC curve or qROC curve (Fig. 5) that gives a performance measure of the DWV along with the false detection avoidance rules. Just like ROC curve it has been plotted as true positive rate (TPR, plotted along the Y-axis) versus false positive rate (FPR, plotted along the $\mathrm{X}$-axis). TPR and FPR must be equal length vectors, with length equal to the number of patients. The $i$ th entry of TPR and FPR, TPR $(i)$ and $\operatorname{FPR}(i)$ respectively correspond the $i$ th patient. $\operatorname{FPR}(i)=1-$ specificity $=1-(\mathrm{TN}(i) / \mathrm{TN}(i)+\mathrm{FP}(i))$ and $\operatorname{TPR}(i)=$ sensitivity $=(\mathrm{TP}(i) / \mathrm{TP}(i)+\mathrm{FN}(i))$, where $\mathrm{TN}(i)$ is the true negative value of the $i$ th patient, etc. For the purpose of the qROC curve plotting we have assigned three false detections to patient 2 in $24 \mathrm{~h}$ (otherwise TPR and FPR will be unequal length vectors), which is the highest in the poll of patients under study (Table II). For a discussion on missing values conventions see [27]. Always the least number of false positives have been chosen (with respect to channel thresholding, see Section V-B and Table II).

For generating the qROC curve we have used the following algorithm called Proc_qROC.

1) Sort FPR in increasing order.

2) Sort each string of repeated entries in FPR in the increasing order of the corresponding TPR values.

3) Plot FPR versus TPR.

4) Select the lowest TPR value and denote it by $L_{T P R}$.

5) Select the highest FPR value and denote it by $\mathrm{H}_{\mathrm{FPR}}$. 
6) Complete the rectangle $\mathrm{R}$ with corner points $\left(0, \mathrm{~L}_{\mathrm{TPR}}\right)$, $(0,1)$ and $\left(\mathrm{H}_{\mathrm{FPR}}, 1\right)$.

7) Include $(0,0)$ as the point of origin.

8) Include $(1,1)$ as the point of termination.

9) The quasi-ROC curve is generated by the lines $\left\{(0,0),\left(0, \mathrm{~L}_{\mathrm{TPR}}\right)\right\}, \quad\left\{\left(0, \mathrm{~L}_{\mathrm{TPR}}\right),\left(\mathrm{L}_{\mathrm{TPR}}, \mathrm{H}_{\mathrm{FPR}}\right)\right\}$, $\left\{\left(\mathrm{L}_{\mathrm{TPR}}, \mathrm{H}_{\mathrm{FPR}}\right),\left(\mathrm{L}_{\mathrm{TPR}}, 1\right)\right\}$ and $\left\{\left(\mathrm{L}_{\mathrm{TPR}}, 1\right),(1,1)\right\}$.

Notice that the shorter are the arms of $\mathrm{R}$ (whose one corner point is $(0,1)$, Fig. 5), the better is the performance of the classifier (each instance has low FPR and high TPR value). This implies that for high performance classifier the area under the qROC curve will be close to 1 . In the case of DWV along with the false detection avoidance measures the area of $\mathrm{R}$ is $(1 / 3) *(1 / 8)=$ $1 / 24$, which indicates that the classifier's performance is very good. The area under the qROC curve is $23 / 24=0.9583$ (Fig. 5).

Note that in case of a qROC curve of a classifier if all the arms of $\mathrm{R}$ (the rectangle on the upper left corner encompassing all the data points) remain quite small the classifier's performance is very good. An R with small arms is a better indicator of classifier performance than the qROC curve with area under the curve close to 1 . Consider the case when $\mathrm{R}$ may have an arm of length close to 1 and another arm with length close to zero, the area under the $\mathrm{qROC}$ curve $=(1-$ area of $\mathrm{R}) \approx 1$. Yet the performance of the classifier is poor, for either the TPR is low or the FPR is high (but not both). In case of DWV along with the false detection avoidance measures the $\mathrm{R}$ associated with the qROC curve has arms length $1 / 3$ and $1 / 8$, i.e., TPR is never below $1-1 / 3=2 / 3$ and FPR is never more than $1 / 8$, which is quite good.

\section{COMPARISON}

Before concluding this paper we briefly want to discuss a comparative study of the DWV algorithm with a difference filter based algorithm described by Qian et al. in [3], which we would like to call as the $Q B B$ algorithm (for a schematic block diagram of the QBB see [3, Fig. 1]). QBB detects sharp transients (STs) in the ECoG signals. Longer duration STs with amplitude higher than certain threshold have been associated with seizure in [3]. See Table V for a technical comparison between the two algorithms. We have implemented the QBB in the following steps (MATLAB code can be downloaded from the first author's website), where $s(n)$ denotes the input ECoG [3].

1) $d(n)=s(n)-s(n-k)$, where $k$ signifies the width of frequency band of interest.

2) $y(n)=d(n) * d(n-m)$, where $m$ is a nonnegative integer.

3) If $|y(n)|>20.5 *$ mean $(|y|)$ holds, where || denotes absolute value and mean $(|y|)$ denotes the mean of the absolute values of $y(n)$ for all $n$, for three successive values of $n$ then all the three values belong to the ST of the input ECoG. $20.5 *$ mean $(|y|)$ is the threshold value (see [3] for the derivation). We have taken first such triplet as the seizure onset (the first point of the triplet) and last such triplet as the seizure offset (the last point of the triplet).

Here there are two parameters in the algorithm, namely, $k$ and $m$. We have taken the values $k=6$ and $m=2$ as in [3]. The performance of the QBB has been summarized in Fig. 6 for
TABLE V

DWV VERSUS QBB

\begin{tabular}{|c|c|}
\hline DWV & QBB \\
\hline $\begin{array}{c}\text { Uses first order temporal } \\
\text { derivative of the EEG to } \\
\text { augment spikes. }\end{array}$ & $\begin{array}{c}\text { Uses product operator of the } \\
\text { EEG to enhance sharp } \\
\text { transients. }\end{array}$ \\
\hline $\begin{array}{c}\text { Uses Gaussian low pass filter. } \\
\text { More effective in presence of } \\
\text { artifacts. }\end{array}$ & $\begin{array}{c}\text { Less effective in presence of } \\
\text { low pass filter. }\end{array}$ \\
\hline $\begin{array}{c}\text { Complimented by false } \\
\text { detection prevention. }\end{array}$ & $\begin{array}{c}\text { Not supported by false } \\
\text { detection prevention. }\end{array}$ \\
\hline $\begin{array}{c}\text { Linear time implementatable } \\
\text { and hence runs in real time. }\end{array}$ & $\begin{array}{c}\text { Quadratic time } \\
\text { implementatable due to the } \\
\text { product operator. }\end{array}$ \\
\hline
\end{tabular}
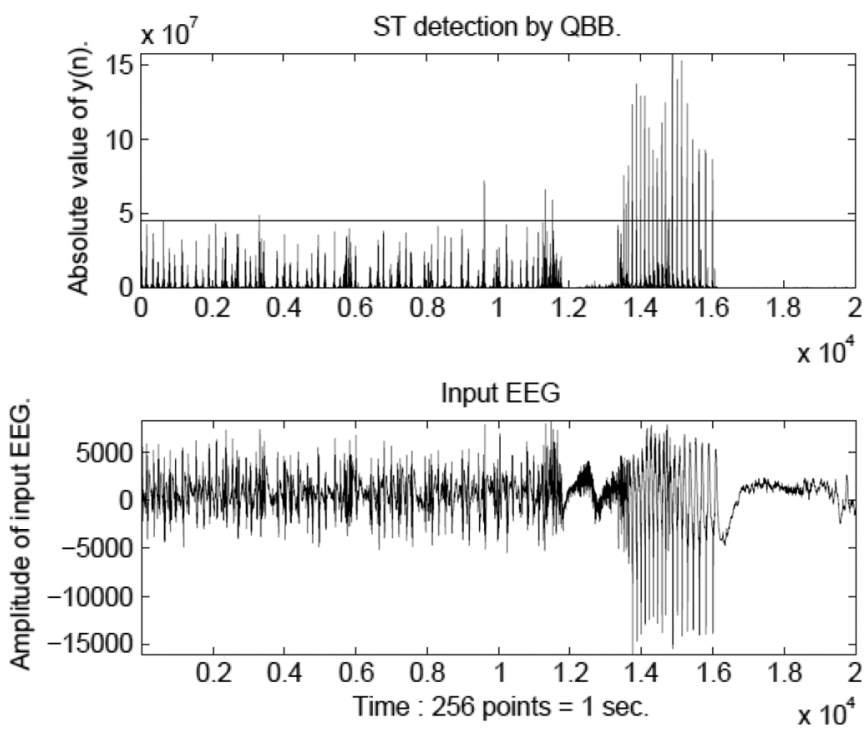

Fig. 6. ST detection by the QBB algorithm on the seventh hour of patient 1 in channel 1-2. A little over $78 \mathrm{~s}$ of recording during a seizure has been presented. Epileptologist identified seizure onset time point is 11100 and offset 16090 , that by DWV are 7302 and 16102 , respectively, and by the QBB are 11522 and 16018 , respectively. The horizontal line in the top panel signifies the threshold.

$78.13 \mathrm{~s}$ of focal electrode ECoG recording of patient 1 during the seventh hour. Channel to channel variation in seizure onset and offset detection is smaller in case of the DWV compared to the QBB. However the false positive rate of seizure detection on nonseizure ECoG by the QBB is very high.

\section{CONCLUSION}

Automatic seizure detection with electronic computers was first reported in the 1970s [28]. In about last 35 years numerous automated detection methodologies have been proposed, but to the best of knowledge of the authors none has turned out to be reliable enough to be used in a clinical setting. A reliable automated seizure detection methodology is also important for intervention at the moment of seizure onset (say by electrical stimulation [29]) or earlier. Therefore research efforts to find new efficient automated detection methodologies are on. In this paper we have proposed a differential operator, normalization and exponentiation based filtering which enhances the seizure part of the ECoG signal and suppresses other artifacts. It operates in linear time and therefore if applied with a linear time 
detection algorithm, such as windowed variance, the whole detection process becomes real-time, what is exactly needed for all practical purposes. An efficient seizure detection algorithm tends to make more false alarms, which erodes its usefulness. To prevent this a battery of eight empirical tests have been devised to verify if seizure has indeed occurred in the detected patch of the continuously monitored signal. Statistical analysis of these false detection prevention methodologies will be a challenging future research.

In this paper we have proposed a novel measure called qROC curve analysis for performance evaluation of a binary classifier, where no probability distribution function is available. In many cases the data set may be too small to infer a distribution function. Moreover epileptic seizures vary widely across patient populations. They may even vary in the same patient over the time. So defining a probability distribution for seizure detectibility by a classifier is an awesome task. To bypass this challenge if a classifier validation methodology is available without relying on probability measures that would be advantageous. A qROC curve exactly does that. A theoretical analysis of qROC curves is beyond the scope of this paper. We will undertake this in future.

In our present work excellent patient specific accuracy has been achieved in detecting seizures with a small number of false detections (Tables II and III) in case of 15 out of 21 patients. The seizure onset detection time has substantially been improved compared to the previous work reported in [10]. Here we have achieved average onset detection delay of $9.2 \mathrm{~s}$ for 15 patients as against $20.45 \mathrm{~s}$ for four patients in [10].

The current method has shown promising success on ECoG, which is relatively noise free, but not artifact free (in fact some patient's data is heavily contaminated with artifacts). In the last section we have compared DWV with a difference operator based ST (and therefore seizure) detection algorithm described in [3], whose false positive rate is very high and channel to chanel difference in onset detection points fluctuate more irratically compared to the DWV. The differential operator can greatly enhance the isolated spikes with respect to the background and therefore may be a potential tool for spike detection in single cell recordings. Since spike detection in electrophysiological data is of predominant importance in neuroscience [30], numerous statistical techniques have been developed for the detection tasks [31]. Our current work shows that differentiation of the signal can improve the outcome of the detection tasks. It can also suppress low frequency artifacts like those generated by body movements and eye blinks. The same is true for low intensity noise.

The algorithm is not specific to brain electrophysiolo-gical signals only. It can be applied to any one dimensional time domain signal to detect abrupt changes, which may be useful for other biomedical signal processing, such as ECG.

\section{ACKNOWLEDGMENT}

The authors are grateful to the Freiburg Seizure Prediction Project, Freiburg, Germany, for generously providing the ECoG data. The authors would also like to thank H. Feldwisch for helping with the data transfer and answering some questions to better understand it. Three anonymous reviewers are being acknowledged for suggesting extensive measures to substantially improve the paper. D. P. Mukherjee and M. Delampady are acknowledged for discussions and helpful suggestions.

\section{REFERENCES}

[1] D. Marr and E. Hildrath, "Theory of edge detection," Proc. R. Soc. London, Series B, Biol. Sci., vol. 207, no. 1167, pp. 187-217, Feb. 1980.

[2] X. Wang, "Laplacian operator-based edge detectors," IEEE Trans. Pattern Anal. Mach. Intell., vol. 29, no. 5, pp. 886-890, May 2007.

[3] J. Qian, J. S. Barlow, and M. P. Beddoes, "A simplified arithmetic detector for EEG sharp transients-preliminary results," IEEE Trans. Biomed. Eng., vol. 35, no. 1, pp. 11-17, Jan. 1988.

[4] A. D. White, P. A. Williams, D. J. Ferraro, S. Clark, S. D. Kadam, F. E. Dudek, and K. J. Staley, "Efficient unsupervised algorithms for the detection of seizures in continuous recordings from rats after brain injury," J. Neurosci. Meth., vol. 152, pp. 256-266, 2006.

[5] J. J. Niederhauser, R. Esteller, J. Echauz, G. Vachtsevanos, and B. Litt, "Detection of seizure precursors from depth-EEG using a sign periodogram transform," IEEE Trans. Biomed. Eng., vol. 50, no. 4, pp. 449-458, Apr. 2003

[6] M. Takigawa, H. Fukuzako, K. Ueyama, K. Takeuchi, T. Fukuzako, and M. Nomaguchi, "Developing of EEG print and its preliminary technical application," Jpn. J. Psychi. Neurol., vol. 48, no. 1, pp. 91-97, 1994.

[7] P. Celka and P. Colditz, "A computer-aided detection of EEG seizures in infants: A singular spectrum approach and performance comparison," IEEE Trans. Biomed. Eng., vol. 49, no. 5, pp. 455-462, May 2002.

[8] V. Krajca, S. Petranek, J. Mohylova, K. Paul, V. Gerla, and L. Lhotska, "Neonatal EEG sleep stages modeling by temporal profiles," in $L N C S$ 4739, R. Moreno-Diaz, Ed. Berlin, Germany: Springer-Verlag, 2007, pp. 195-201.

[9] N. Paivinen, S. Lammi, A. Pitkanen, J. Nissinen, M. Penttonen, and T. Gronfors, "Epileptic seizure detection: A nonlinear viewpoint," Comp. Meth. Progr. Biomed., vol. 79, no. 2, pp. 151-159, 2005.

[10] K. Majumdar, "Differential operator in seizure detection," Patt. Recog. Lett. 2010 [Online]. Available: http://arxiv.org/ftp/arxiv/papers/0911/ 0911.0188.pdf

[11] D.-M. Ward, R. D. Jones, P. J. Bones, and G. J. Carroll, "Enhancement of deep epileptiform activity in the EEG via 3-D adaptive spatial filtering," IEEE Trans. Biomed. Eng., vol. 46, no. 6, pp. 707-716, Jun. 1999.

[12] T. O'Haver, Introduction to signal processing: Differentiation [Online]. Available: http://terpconnect.umd.edu/ toh/SPECTRUM/Differentiation.html

[13] P. E. McSharry, T. He, L. A. Smith, and L. Tarassenko, "Linear and non-linear methods for automatic seizure detection in scalp electro-encephalogram recordings," Med. Biol. Eng. Comp., vol. 40, pp. 447-461, 2002.

[14] P. E. McSharry, L. A. Smith, and L. Tarassenko, "Comparison of predictability of epileptic seizures by a linear and a nonlinear method," IEEE Trans. Biomed. Eng., vol. 50, no. 5, pp. 628-633, May 2003.

[15] D. P. Mukherjee, private communication. Sep. 2010.

[16] K. Majumdar, "Constraining minimum norm inverse by phase synchronization and signal power of the scalp EEG channels," IEEE Trans. Biomed. Eng., vol. 56, no. 4, pp. 1228-1237, Apr. 2009.

[17] T. M. Apostol, Mathematical Analysis, 2nd ed. Reading, MA: Addison Wesley Longman, 1974.

[18] Freiburg Seizure Prediction Project. Freiburg, Germany [Online]. Available: https://epilepsy.uni-freiburg.de/, 2008

[19] R. Aschenbrenner-Scheibe, T. Maiwald, M. Winterhalder, H. U. Voss, J. Timmer, and A. Schulze-Bonhage, "How well can epileptic seizures be predicted? An evaluation of a nonlinear method," Brain, vol. 126, pp. 2616-2626, 2003.

[20] T. Maiwald, M. Winterhalder, R. Aschenbrenner-Scheibe, H. U. Voss, A. Schulze-Bonhage, and J. Timmer, "Comparison of three nonlinear seizure prediction methods by means of the seizure prediction characteristics," Physica D, vol. 194, pp. 357-368, 2004.

[21] M. Winterhalder, J. Schelter, T. Maiwald, A. Brandt, A. Schad, A. Schulze-Bonhage, and J. Timmer, "Spatio-temporal patient-individual assessment of synchronization changes for epileptic seizure prediction," Clin. Neurophysiol., vol. 117, pp. 2399-2413, 2006. 
[22] H. C. Lee, W. van Drongelen, A. B. McGee, D. M. Frim, and M. H. Kohrman, "Comparison of seizure detection algorithms in continuously monitoring pediatric patients," J. Clin. Neurophysiol., vol. 24, no. 2, pp. 137-146, Apr. 2007.

[23] H. Qu and J. Gotman, "A patient-specific algorithm for the detection of seizure onset in long-term EEG monitoring: Possible use as a warning device," IEEE Trans. Biomed. Eng., vol. 44, no. 2, pp. 115-122, Feb. 1997.

[24] M. Tito, M. Cabrerio, M. Ayala, P. Jayakar, and M. Adjouadi, "Seizure detection: An assessment of time and frequency-based features in a unified two-dimensional decisional space using nonlinear decision functions," J. Clin. Neurophysiol., vol. 26, no. 6, pp. 381-391, Dec. 2009.

[25] T. Fawcett, "An introduction to ROC analysis," Pat. Recog. Lett., vol. 27, pp. 861-874, 2006.

[26] W. Chaovalitwongse, L. D. Iasemidis, P. M. Pardalos, P. R. Carney, D.-S. Shiau, and J. C. Sackellares, "Performance of a seizure warning algorithm based on the dynamics of intracranial EEG," Epilepsy Res., vol. 64 , pp. 93-113, 2005 .

[27] W. J. Krzanowski and D. J. Hand, ROC Curves for Continuous Data. Boca Raton: CRC Press, 2009.

[28] J. Gotman and P. Gloor, "Automatic recognition and quantification of interictal epileptic activity in the human scalp EEG," Electroencephalogra. Clin. Neurophysiol., vol. 41, no. 5, pp. 513-529, Nov. 1976.

[29] D. M. Durand and M. Bikson, "Suppression and control of epileptiform activity by electrical stimulation: A review," Proc. IEEE, vol. 89, no. 7, pp. 1065-1082, Jul. 2001.

[30] R. Q. Quiroga, L. Reddy, G. Kreiman, C. Koch, and I. Fried, "Invariant visual representation by single neurons in the human brain," Nature, vol. 435, pp. 1102-1107, Jun 2005.

[31] E. N. Brown, R. E. Kass, and P. P. Mitra, "Multiple neural spike train data analysis: State-of-the-art and future challenges," Nat. Neurosci., vol. 7, no. 5, pp. 456-461, Apr. 2004.

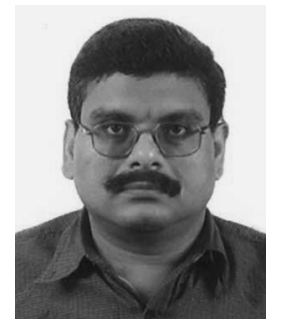

Kaushik K. Majumdar (M'06-SM'10) received the B.Sc. degree from the University of Calcutta, Calcutta, India, in 1990, the M.Sc. degree in mathematics from the Annamalai University, Annamalai Nagar, India, 1996, and the M.Tech. and Ph.D. degrees in computer science from the Indian Statistical Institute, Calcutta, India, in 1999 and 2003 , respectively.

He was a Research Associate with the Council of Scientific and Industrial Research of the Government of India (2003-2004) and a Fulbright Fellow in the Department of Computer Science of the University of Memphis, Memphis, TN (2005-2006). He worked jointly as an R\&D Scientist in the Electrical Geodesics Inc., and as a Research Associate in the University of Oregon, Eugene (2006). He was a postdoctoral fellow in the Institute of Mathematical Sciences in Chennai, India (2006-2007). Until August 2008 he worked as an Invited Scientist in the Odyssee Group in INRIA Sophia Antipolis, France. He also worked as a postdoctoral fellow in the Center for Complex Systems and Brain Sciences in the Florida Atlantic University, Boca Raton, in 2008-2009. Since May 2009 he is an Assistant Professor in the Indian Statistical Institute, Bangalore Center. So far he has authored more than 25 papers in international journals and conferences. His interests are in neural signal processing, mathematical and computational modeling of the brain functions and nonlinear system theoretic studies of epilepsy.

Dr. Majumdar is a member of the American Mathematical Society. He is a recipient of the Norbert Wiener outstanding paper award with D. Dutta Majumder in 2005 for their paper in Kybernetes in 2004. His name has been featuring in Marquis Whose Who of the World since 2007.

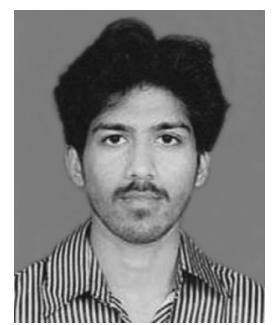

Pratap Vardhan was born in Hyderabad, India, on April 28, 1989. He is currently an undergraduate student in the Department of Electronics and Communication, Maulana Azad National Institute of Technology, Bhopal, India.

His current research interests include signal processing, image processing, spatial analysis and human-computer interaction. 